\title{
Fusion Estimation Algorithm with Uncertain Noises and Its Application in Navigation System
}

\author{
Zhiping Ren ${ }^{1}$ and Huili Wang ${ }^{2}$ \\ ${ }^{1}$ School of Electronic Engineering, Xian Shiyou University, Xi'an, Shaanxi 710065, China \\ ${ }^{2}$ School of Automation, Northwestern Polytechnical University, Xian, Shaanxi 710072, China \\ Correspondence should be addressed to Zhiping Ren; renzp@xsyu.edu.cn
}

Received 30 April 2014; Revised 9 September 2014; Accepted 9 September 2014

Academic Editor: Moran Wang

Copyright (C) 2015 Z. Ren and H. Wang. This is an open access article distributed under the Creative Commons Attribution License, which permits unrestricted use, distribution, and reproduction in any medium, provided the original work is properly cited.

\begin{abstract}
In order to solve the problem of uncertain noise during the measurement of actual system, an extended Kalman filter fusion estimation method based on multisensor fusion algorithm with uncertain effects is proposed. Then the equivalent measurement and the corresponding error matrix are estimated by the proposed uncertain fusing algorithm. Submit the results into the system model for filter processing and the optimal estimation can be obtained by the filtering method. Finally, the algorithm is verified in the GPS/INS navigation system which shows that the fusion result with uncertainty effect is much better than then fusion result with independent noise due to the consideration of correlated noise and uncertain effects for the actual system. This is also validates the effectiveness and practicality of the proposed algorithm.
\end{abstract}

\section{Introduction}

In order to improve the accuracy of vehicle positioning, multiple sensors are installed on the vehicle to overcome the defect of single sensor. For instance, global positioning system (GPS) is a satellite-based navigation system and can provide accurate three-dimensional information on the position and velocity. However, the lack of credibility and nonautonomous of GPS in some cases, often leads to blind field and cannot be effectively positioned. Inertial navigation system (INS) is a standalone system that provides linear acceleration and angular velocity at high speed, but as time goes by it significantly reduces its accuracy, position, and velocity and error rate information increased. The combination of GPS and INS can provide accuracy position and enhance the reliability of the system, and therefore has been widely applied in the integrated navigation system [1-3].

The Kalman filtering (KF) approach is one of the widely used methods for the navigation system $[4,5]$. However, KF is the optimal estimation of minimum mean square error for a linear time invariant system and has a strict requirement for the process noise covariance and measurement noise covariance. Based on the KF, other methods like particle filter, cubature Kalman filter (CKF), and extended Kalman particle filter are also used in navigation [6,7]. Arasaratnam et al. studied the continuous discrete system by the cubature KF [6]. However the statistical characteristics of noise should be decided when using filter method and therefore many studies assumed that the measurement noise and system noise are independent white noise [8-10]. Cappelle et al. presented an algorithm of vehicle positioning based on Kalman filter, which integrated data from INS and GPS with the assumption that the system noise and measurement noise are independent white noise [11]. Carlson presented the famous federated square root filter which assumes the initial estimation error cross-covariance matrices among the local subsystems to be zero [12]. Deng and Qi proposed a fusion algorithm weighted by scalars with the assumption that the state estimation errors among the local subsystems to be uncorrelated and simplify the calculation [13]. While the ideal assumption, which local estimators are with independent or uncorrelated measurement noise, does not accord with the general case, and sometimes can cause bias and divergence problem due to cumulative errors for the actual system.

In order to better describe the actual system, some scholars have studied the fusion estimation with the correlated 
noise $[14,15]$. Giremus et al. study the correlated noise for GPS navigation by comparing two KF methods [14]. Based on the work [13], Sun studied the correlation among local estimation errors and proposed a multisensor optimal information fusion algorithm weighted by matrices in the linear minimum variance sense [16, 17]. An optimal fusion algorithm using general weighting matrices for more than two local correlated estimates was proposed by Hong et al. [18], where the global state estimate was a linear combination of all the local state estimates, and this required a large amount of calculation. To reduce the amount of calculations, Qiu et al. studied a diagonal weighting matrices method for fusion of local estimates [19]; however it introduced a loss in estimation accuracy. The present fusion methods for the navigation system are mainly focused on the independent noise and correlated noises and do not conclude the uncertain factors during the measurement for the actual system.

In practice, when GPS/INS and other sensors are installed on the vehicle to navigate, the measurement noise is not only correlated, but also there exists some uncertainty effects. For instance, the common environment, like the vibration of the platform (vehicle) during the driving, will cause the measurements noise from all sensors installed on it to be intercorrelated. What is more, the not well-informed statistical characteristics of measurement noise and the transformation error of different coordinate systems causing uncertainty factors do exist in the actual system. Sometimes, the uncertainty will significantly affect the accuracy of the fusion estimation. This means that the filter method cannot be used directly for the actual system when considering the uncertainty factors during the measurement.

This paper presents an extended Kalman filter fusion estimation method based on multisensor fusion algorithm with uncertainty effect. After dealing with the measurement information of multiple sensors, the equivalent measurement is obtained, together with the corresponding error matrix. Using the extended Kalman filtering, the exact position will be obtained.

The outline of the remainder of the paper is as follows. In Section 2, the system model is given and the extended Kalman filter function is briefly introduced. The uncertainty fusion estimation algorithm is the main subject of Section 3. In Section 4, results from the field tests are presented to illustrate the effectiveness of the algorithm proposed in Section 3. Section 5 contains a summary and conclusions.

\section{System Model}

Consider the stochastic system with multiple sensors:

$$
\begin{aligned}
\dot{\mathbf{x}}(t) & =\mathbf{f}[\mathbf{x}(t), \mathbf{u}(t), \mathbf{w}(t), t] \\
\mathbf{z}_{i}(k) & =\mathbf{h}_{i}(\mathbf{x}(k))+\mathbf{v}_{i}(k) \quad(i=1,2, \ldots, m),
\end{aligned}
$$

where, $\mathbf{x}$ is the state vector, $\mathbf{w}$ is the system noise, $\mathbf{z}_{i}(k)(i=$ $1,2, \ldots, m)$ the measurement vector of sensor $i$ and $\mathbf{v}_{i}(k)$ is the corresponding noise, $\mathbf{f}(\cdot)$ and $\mathbf{h}_{i}(\cdot)$ are the continuous function, denote the state function and measurement function, and $\mathbf{u}$ is the input vector.
Different sensors, installed on the same platform, share the same environment which makes the measurements noise $\mathbf{v}_{i}(k)$, are intercorrelated, and uncertainty factors exist for the vibration of the vehicle during the driving. Therefore the statistics characteristics should be decided before using extended KF on system (1). After the fusion processing of the measurement information, the system (1) can be rewritten as

$$
\begin{aligned}
\dot{\mathbf{x}}(t) & =\mathbf{f}[\mathbf{x}(t), \mathbf{w}(t), t] \\
\mathbf{z}(k) & =\mathbf{h}(\mathbf{x}(k))+\mathbf{v}(k),
\end{aligned}
$$

where, $\mathbf{z}(k)$ is the equivalent measurement obtained by uncertain fusion estimation algorithm, and $\mathbf{v}(k)$ is the corresponding error with zero mean and variance $\mathbf{R}(k)$. Discrete system (2), the extended Kalman filter algorithm is

$$
\begin{aligned}
\widehat{\mathbf{x}}(k \mid k)= & \widehat{\mathbf{x}}(k \mid k-1)+\mathbf{K}(k)[\mathbf{z}(k)-\mathbf{h}(\widehat{\mathbf{x}}(k \mid k-1))] \\
\mathbf{K}(k)= & \mathbf{P}(k \mid k-1) \mathbf{H}(k)^{T} \\
& \times\left[\mathbf{H}(k) \mathbf{P}(k \mid k-1) \mathbf{H}(k)^{T}+\mathbf{R}(k)\right]^{-1} \\
\mathbf{P}(k \mid k)= & {[\mathbf{I}-\mathbf{K}(k) \mathbf{H}(k)] \mathbf{P}(k \mid k-1) } \\
\widehat{\mathbf{x}}(k \mid k-1)= & \mathbf{f}(\widehat{\mathbf{x}}(k-1 \mid k-1)) \\
\mathbf{P}(k \mid k-1)= & \mathbf{F}(k, k-1) \mathbf{P}(k-1 \mid k-1) \mathbf{F}(k, k-1)^{T} \\
& +\mathbf{Q}(k-1),
\end{aligned}
$$

where, $k$ denote the discrete time, $\widehat{\mathbf{x}}(k \mid k)$ is the estimator of state vector $\mathbf{x}$ at time $k, \widehat{\mathbf{x}}(k \mid k-1)$ is the predictor of $\widehat{\mathbf{x}}(k-1 \mid$ $k-1), \mathbf{z}(k)$ is the measurement value at time $k, \mathbf{K}(k)$ denotes the Kalman gain matrix, $\mathbf{P}(k \mid k-1)$ and $\mathbf{P}(k \mid k)$ are the MSE matrix of $\widehat{\mathbf{x}}(k \mid k-1)$ and $\widehat{\mathbf{x}}(k \mid k)$, I denote the identity matrix, and $\mathbf{F}(k, k-1)=\partial \mathbf{f} /\left.\partial \mathbf{x}\right|_{\widehat{\mathbf{x}}(k-1 \mid k-1)}, \mathbf{H}(k)=\partial \mathbf{h} /\left.\partial \mathbf{x}\right|_{\widehat{\mathbf{x}}(k \mid k-1)}$, and $\mathbf{R}(k), \mathbf{Q}(k)$ are the covariance matrix of measurement system noise.

\section{Uncertain Fusion Estimation Algorithm}

Theory. Suppose that a multisensor system consists of $m$ sensors, and the system noise is composed of correlated noise and uncertainty term. The measurement information obtained from the $i$ th sensor at time $k$ is denoted:

$$
\mathbf{z}_{i}(k)=\mathbf{z}(k)+\mathbf{v}_{i}(k)+\Delta \mathbf{z}_{i}(k) \quad i=1,2, \ldots, m,
$$

where, $\mathbf{z}(k) \in R^{n}$ denotes physical vector to be estimated, $\mathbf{z}_{i}(k) \in R^{n},(i=1,2, \ldots, m)$ is the measurement value from $i$ th sensor at time $k$, corresponding measurement error is $\mathbf{v}_{i}(k) \in R^{n},(i=1,2, \ldots, m)$ with zero mean and the cross-covariance matrices $\mathbf{R}_{i j}(k) \in R^{n \times n}, \Delta \mathbf{z}_{i}(k) \in R^{n}$, and $(i=1,2, \ldots, m)$ is the uncertainty term. Then the fusion estimation result $\widehat{\mathbf{z}}(k)$ and corresponding error covariance matrix $\mathbf{R}(k)$ are given by the following: 


$$
\widehat{\mathbf{z}}(k)=\sum_{i=1}^{m} \boldsymbol{\Lambda}_{i}(k) \mathbf{z}_{i}(k)
$$

$\mathbf{R}(k)$

$$
=\sum_{i=1}^{m} \sum_{j=1}^{m} \boldsymbol{\Lambda}_{i}(k)\left[a_{i j} \mathbf{A}(k)+b_{i j} \mathbf{R}_{i i}(k)+c_{i j} \mathbf{R}_{j j}(k)\right] \boldsymbol{\Lambda}_{j}^{T}(k),
$$

where $\widehat{\mathbf{z}}(k)$ is fusion estimation, $\mathbf{R}(k)$ is the corresponding error covariance matrix, $a_{i j}>0$, and $b_{j i}>0, c_{i j}>0$ are positive real numbers. $\mathbf{A}(k)=\sup _{i=1,2, \ldots, m} E\left(\Delta \mathbf{z}_{i}(k) \Delta \mathbf{z}_{i}^{T}(k)\right)$ is supremum of uncertainty error, $E$ is the expectation, and the superscript $T$ denotes the transpose. $\Lambda_{1}(k), \Lambda_{2}(k), \ldots, \Lambda_{m}(k)$ are coefficient matrix, which can be calculated by the following:

$\mathbf{S}(k)$

$$
\begin{aligned}
& {\left[\begin{array}{llll}
\boldsymbol{\Lambda}_{1}(k) & \Lambda_{2}(k) & \cdots & \Lambda_{m-1}(k)
\end{array}\right]=\mathbf{W}(k) \mathbf{S}^{-1}(k),} \\
& \boldsymbol{\Lambda}_{m}(k)=\mathbf{I}-\sum_{i=1}^{m-1} \boldsymbol{\Lambda}_{i}(k), \\
& \mathbf{W}(k)=\left[\begin{array}{lllll}
\mathbf{T}_{m m} & (k)-\mathbf{T}_{1 m}(k) \mathbf{T}_{m m}(k)-\mathbf{T}_{2 m}(k) \cdots \mathbf{T}_{m m}(k)-\mathbf{T}_{m-1, m}(k)
\end{array}\right],
\end{aligned}
$$

$$
\begin{aligned}
& {\left[\begin{array}{cccc}
\mathbf{S}_{11}(k) & \mathbf{S}_{12}(k) & \cdots & \mathbf{S}_{1, m-1}(k) \\
\mathbf{S}_{21}^{T}(k) & \mathbf{S}_{22}(k) & \vdots & \mathbf{S}_{2, m-1}(k) \\
\vdots & \vdots & \ddots & \vdots \\
\mathbf{S}_{m-1,1}^{T}(k) & \mathbf{S}_{m-1,2}^{T}(k) & \cdots & \mathbf{S}_{m-1, m-1}(k)
\end{array}\right] } \\
= & {\left[\begin{array}{cccc}
\mathbf{T}_{11}(k)-\mathbf{T}_{1 m}(k)-\mathbf{T}_{m 1}(k)+\mathbf{T}_{m m}(k) & \mathbf{T}_{12}(k)-\mathbf{T}_{1 m}(k)-\mathbf{T}_{m 2}(k)+\mathbf{T}_{m m}(k) & \cdots & \mathbf{T}_{1, m-1}(k)-\mathbf{T}_{1 m}(k)-\mathbf{T}_{m, m-1}(k)+\mathbf{T}_{m m}(k) \\
\mathbf{T}_{21}(k)-\mathbf{T}_{2 m}(k)-\mathbf{T}_{m 1}(k)+\mathbf{T}_{m m}(k) & \mathbf{T}_{22}(k)-\mathbf{T}_{2 m}(k)-\mathbf{T}_{m 2}(k)+\mathbf{T}_{m m}(k) & \cdots & \mathbf{T}_{2, m-1}(k)-\mathbf{T}_{2 m}(k)-\mathbf{T}_{m, m-1}(k)+\mathbf{T}_{m m}(k) \\
\vdots & \vdots & \ddots & \vdots \\
\mathbf{T}_{m-1,1}(k)-\mathbf{T}_{m-1, m}(k)-\mathbf{T}_{m 1}(k)+\mathbf{T}_{m m}(k) & \mathbf{T}_{m-1,1}(k)-\mathbf{T}_{m-1, m}(k)-\mathbf{T}_{m 2}(k)+\mathbf{T}_{m m}(k) & \cdots & \mathbf{T}_{m-1, m-1}(k)-\mathbf{T}_{m-1, m}(k)-\mathbf{T}_{m, m-1}(k)+\mathbf{T}_{m m}(k)
\end{array}\right] } \\
& \\
& \mathbf{T}_{i j}(k)=a_{i j} \mathbf{A}(k)+b_{i j} \mathbf{R}_{i i}(k)+c_{i j} \mathbf{R}_{j j}(k),
\end{aligned}
$$

where $\mathbf{R}_{i j}(k)(i, j=1,2, \ldots, m)$ are the cross-covariance of $\mathbf{v}_{i}(k), \mathbf{v}_{j}(k)(i, j=1,2, \ldots, m)$ and the value of $a_{i j}, b_{i j}, c_{i j}$ and $\mathbf{A}(k)$ are determined by the statistical characteristics of the actual system and the accuracy of the sensors.

Proof. Let $\mathbf{z}(k) \in R^{n}$ is the physical vector to be estimated; $\mathbf{z}_{i}(k) \in R^{n}(i=1,2, \ldots, m)$ denote observation value from the $i$ th sensor at time $k, \mathbf{v}_{i}(k) \in R^{n},(i=1,2, \ldots, m)$ is the corresponding measurement error, which is assumed to be zero mean, covariance matrix is $\mathbf{R}_{i i}(k) \in R^{n \times n}$, and $\Delta \mathbf{z}_{i}(k) \in R^{n}$ denotes uncertainty term from the $i$ th sensor; the measurement can be written as

$$
\mathbf{z}_{i}(k)=\mathbf{z}(k)+\mathbf{v}_{i}(k)+\Delta \mathbf{z}_{i}(k) \quad i=1,2, \ldots, m .
$$

Define the fusion estimator as

$$
\widehat{\mathbf{z}}(k)=\sum_{i=1}^{m} \Lambda_{i}(k) \mathbf{z}_{i}(k)
$$

where, $\widehat{\mathbf{z}}(k)$ denotes fusion result and $\boldsymbol{\Lambda}_{1}(k), \boldsymbol{\Lambda}_{2}(k), \ldots$, $\Lambda_{m}(k) \in R^{n \times n}$ are weighting matrices to be determined. It is well known that a global optimal estimate should be unbiased and the error covariance matrix is minimum. From the assumption of an unbiased condition, the constraints of the respective weights should satisfy

$$
\sum_{i=1}^{m} \boldsymbol{\Lambda}_{i}(k)=\mathbf{I}
$$

Therefore the error covariance matrix is

$$
\begin{aligned}
\mathbf{R}(k)=E[ & \left(\sum_{i=1}^{m} \boldsymbol{\Lambda}_{i}(k)\left(\mathbf{v}_{i}(k)+\Delta \mathbf{z}_{i}(k)\right)\right) \\
& \left.\times\left(\sum_{j=1}^{m} \boldsymbol{\Lambda}_{j}(k)\left(\mathbf{v}_{j}(k)+\Delta \mathbf{z}_{j}(k)\right)\right)^{T}\right] \\
=E( & \sum_{i=1}^{m} \sum_{j=1}^{m} \boldsymbol{\Lambda}_{i}(k) \Delta \mathbf{z}_{i}(k) \Delta \mathbf{z}_{j}^{T}(k) \boldsymbol{\Lambda}_{j}^{T}(k) \\
& +\sum_{i=1}^{m} \sum_{j=1}^{m} \boldsymbol{\Lambda}_{i}(k) \Delta \mathbf{z}_{i}(k) \mathbf{v}_{j}^{T}(k) \boldsymbol{\Lambda}_{j}^{T}(k) \\
& +\sum_{i=1}^{m} \sum_{j=1}^{m} \boldsymbol{\Lambda}_{i}(k) \mathbf{v}_{i}(k) \Delta \mathbf{z}_{j}^{T}(k) \boldsymbol{\Lambda}_{j}^{T}(k) \\
& \left.+\sum_{i=1}^{m} \sum_{j=1}^{m} \boldsymbol{\Lambda}_{i}(k) \mathbf{v}_{i}(k) \mathbf{v}_{j}^{T}(k) \boldsymbol{\Lambda}_{j}^{T}(k)\right)
\end{aligned}
$$




$$
\begin{aligned}
=\sum_{i=1}^{m} \sum_{j=1}^{m} \boldsymbol{\Lambda}_{i}(k)[ & E\left(\Delta \mathbf{z}_{i}(k) \Delta \mathbf{z}_{j}^{T}(k)\right) \\
& +E\left(\Delta \mathbf{z}_{i}(k) \mathbf{v}_{j}^{T}(k)\right) \\
& +E\left(\mathbf{v}_{i}(k) \Delta \mathbf{z}_{j}^{T}(k)\right) \\
& \left.+E\left(\mathbf{v}_{i}(k) \mathbf{v}_{j}^{T}(k)\right)\right] \boldsymbol{\Lambda}_{j}^{T}(k) .
\end{aligned}
$$

For any real number $\lambda_{i j} \neq 0, \lambda_{j i} \neq 0$, and $\mu_{i j} \neq 0$, the following inequality is true:

$$
\begin{gathered}
E\left[\left(\lambda_{i j} \Delta \mathbf{z}_{i}(k)-\frac{\mathbf{v}_{j}(k)}{\lambda_{i j}}\right)\left(\lambda_{i j} \Delta \mathbf{z}_{i}(k)-\frac{\mathbf{v}_{j}(k)}{\lambda_{i j}}\right)^{T}\right] \geq \mathbf{0}, \\
E\left[\left(\lambda_{j i} \Delta \mathbf{z}_{j}(k)-\frac{\mathbf{v}_{i}(k)}{\lambda_{j i}}\right)\left(\lambda_{j i} \Delta \mathbf{z}_{j}(k)-\frac{\mathbf{v}_{i}(k)}{\lambda_{j i}}\right)^{T}\right] \geq \mathbf{0}, \\
E\left[\left(\mu_{i j} \Delta \mathbf{z}_{i}(k)-\frac{\Delta \mathbf{z}_{j}(k)}{\mu_{i j}}\right)\left(\mu_{i j} \Delta \mathbf{z}_{i}(k)-\frac{\Delta \mathbf{z}_{j}(k)}{\mu_{i j}}\right)^{T}\right] \geq \mathbf{0} .
\end{gathered}
$$

Simplify the above equation, then

$$
\begin{aligned}
& E\left(\Delta \mathbf{z}_{i}(k) \mathbf{v}_{j}^{T}(k)\right)+E\left(\mathbf{v}_{j}(k) \Delta \mathbf{z}_{i}^{T}(k)\right) \\
& \leq \lambda_{i j}^{2} E\left(\Delta \mathbf{z}_{i}(k) \Delta \mathbf{z}_{i}^{T}(k)\right)+\frac{E\left(\mathbf{v}_{j}(k) \mathbf{v}_{j}^{T}(k)\right)}{\lambda_{i j}} \\
& =\lambda_{i j}^{2} E\left(\Delta \mathbf{z}_{i}(k) \Delta \mathbf{z}_{i}^{T}(k)\right)+\frac{\mathbf{R}_{j j}(k)}{\lambda_{i j}^{2}}, \\
& E\left(\Delta \mathbf{z}_{j}(k) \mathbf{v}_{i}^{T}(k)\right)+E\left(\mathbf{v}_{i}(k) \Delta \mathbf{z}_{j}^{T}(k)\right) \\
& \leq \lambda_{j i}^{2} E\left(\Delta \mathbf{z}_{j}(k) \Delta \mathbf{z}_{j}^{T}(k)\right)+\frac{E\left(\mathbf{v}_{i}(k) \mathbf{v}_{i}^{T}(k)\right)}{\lambda_{j i}^{2}} \\
& =\lambda_{j i}^{2} E\left(\Delta \mathbf{z}_{j}(k) \Delta \mathbf{z}_{j}^{T}(k)\right)+\frac{\mathbf{R}_{i i}(k)}{\lambda_{j i}^{2}}, \\
& E\left(\Delta \mathbf{z}_{i}(k) \Delta \mathbf{z}_{j}^{T}(k)\right)+E\left(\Delta \mathbf{z}_{j}(k) \Delta \mathbf{z}_{i}^{T}(k)\right) \\
& \leq \mu_{i j}^{2} E\left(\Delta \mathbf{z}_{i}(k) \Delta \mathbf{z}_{i}^{T}(k)\right)+\frac{E\left(\Delta \mathbf{z}_{j}(k) \Delta \mathbf{z}_{j}^{T}(k)\right)}{\mu_{i j}^{2}} .
\end{aligned}
$$

Define $\mathbf{A}(k)=\sup _{i=1,2, \ldots, m} E\left(\Delta \mathbf{z}_{i}(k) \Delta \mathbf{z}_{i}^{T}(k)\right)$ and denote the supremum of the uncertainty error. Combine (15), then

$$
\begin{aligned}
E\left(\Delta \mathbf{z}_{i}(k) \Delta \mathbf{z}_{j}^{T}(k)\right)+E\left(\Delta \mathbf{z}_{i}(k) \mathbf{v}_{j}^{T}(k)\right) \\
+E\left(\mathbf{v}_{i}(k) \Delta \mathbf{z}_{j}^{T}(k)\right)+E\left(\mathbf{v}_{i}(k) \mathbf{v}_{j}^{T}(k)\right)
\end{aligned}
$$

$$
\begin{gathered}
\leq \frac{\lambda_{i j}^{2}+\mu_{i j}^{2}}{2 \cdot E\left(\Delta \mathbf{z}_{i}(k) \Delta \mathbf{z}_{i}^{T}(k)\right)} \\
+\frac{\mathbf{R}_{i i}(k)}{2 \lambda_{j i}^{2}}+\frac{\mathbf{R}_{j j}(k)}{2 \lambda_{i j}^{2}} \\
+\frac{\lambda_{j i}^{2}+1 / \mu_{i j}^{2}}{2 \cdot E\left(\Delta \mathbf{z}_{j}(k) \Delta \mathbf{z}_{j}^{T}(k)\right)} .
\end{gathered}
$$

Let $a_{i j}=\left(\lambda_{i j}^{2}+\lambda_{j i}^{2}+\mu_{i j}^{2}+1 / \mu_{i j}^{2}\right) / 2, b_{i j}=1 /\left(2 \lambda_{j i}^{2}\right)$, $c_{i j}=1 /\left(2 \lambda_{i j}^{2}\right)$, and $\mathbf{T}_{i j}(k)=a_{i j} \mathbf{A}(k)+b_{i j} \mathbf{R}_{i i}(k)+c_{i j} \mathbf{R}_{j j}(k)$, It is obvious that

$$
\begin{gathered}
a_{i j}>0, \quad b_{j i}>0, \quad c_{i j}>0 \\
\mathbf{R}(k) \leq \sum_{i=1}^{m} \sum_{j=1}^{m} \boldsymbol{\Lambda}_{i}(k) \mathbf{T}_{i j}(k) \boldsymbol{\Lambda}_{j}^{T}(k) .
\end{gathered}
$$

Let $\mathbf{P}(k)=\sum_{i=1}^{m} \sum_{j=1}^{m} \Lambda_{i}(k) \mathbf{T}_{i j}(k) \boldsymbol{\Lambda}_{j}^{T}(k)$, then

$$
\begin{aligned}
\mathbf{P}(k)= & \sum_{i=1}^{m-1} \sum_{j=1}^{m-1} \boldsymbol{\Lambda}_{i}(k) \mathbf{T}_{i j}(k) \boldsymbol{\Lambda}_{j}^{T}(k) \\
& +\sum_{i=1}^{m-1} \boldsymbol{\Lambda}_{i}(k) \mathbf{T}_{i m}(k)\left[\mathbf{I}-\sum_{i=1}^{m-1} \boldsymbol{\Lambda}_{i}(k)\right]^{T} \\
& +\sum_{i=1}^{m-1}\left[\mathbf{I}-\sum_{i=1}^{m-1} \boldsymbol{\Lambda}_{i}(k)\right] \mathbf{T}_{m j}(k) \boldsymbol{\Lambda}_{j}^{T}(k) \\
& +\left[\mathbf{I}-\sum_{i=1}^{m-1} \boldsymbol{\Lambda}_{i}(k)\right] \mathbf{T}_{m m}(k)\left[\mathbf{I}-\sum_{i=1}^{m-1} \boldsymbol{\Lambda}_{i}(k)\right]^{T} .
\end{aligned}
$$

Let $\partial \operatorname{tr}(\mathbf{P}(k)) / \partial \boldsymbol{\Lambda}_{k}(k)=\mathbf{0}(k=1,2, \ldots, m-1),(\operatorname{tr}$ denotes trace of the matrix), then

$$
\begin{array}{r}
\sum_{i=1}^{m-1} \Lambda_{i}(k)\left[\mathbf{T}_{i l}(k)-\mathbf{T}_{i m}(k)-\mathbf{T}_{m l}(k)+\mathbf{T}_{m m}(k)\right] \\
=\mathbf{T}_{m m}(k)-\mathbf{T}_{l m}(k) \quad(l=1,2, \ldots, m-1)
\end{array}
$$

The matrix form of (19) can be written as

$$
\left[\begin{array}{llll}
\Lambda_{1}(k) & \Lambda_{2}(k) & \cdots & \Lambda_{m-1}(k)
\end{array}\right]=\mathbf{W}(k) \mathbf{S}^{-1}(k),
$$

where 


$$
\begin{aligned}
& \mathbf{W}(k)=\left[\begin{array}{llll}
\mathbf{T}_{m m}(k)-\mathbf{T}_{1 m}(k) & \mathbf{T}_{m m}(k)-\mathbf{T}_{2 m}(k) & \cdots & \mathbf{T}_{m m}(k)-\mathbf{T}_{m-1, m}(k)
\end{array}\right], \\
& \mathbf{S}(k) \\
& =\left[\begin{array}{cccc}
\mathbf{S}_{11}(k) & \mathbf{S}_{12}(k) & \cdots & \mathbf{S}_{1, m-1}(k) \\
\mathbf{S}_{21}^{T}(k) & \mathbf{S}_{22}(k) & \vdots & \mathbf{S}_{2, m-1}(k) \\
\vdots & \vdots & \ddots & \vdots \\
\mathbf{S}_{m-1,1}^{T}(k) & \mathbf{S}_{m-1,2}^{T}(k) & \cdots & \mathbf{S}_{m-1, m-1}(k)
\end{array}\right] \\
& =\left[\begin{array}{cccc}
\mathbf{T}_{11}(k)-\mathbf{T}_{1 m}(k)-\mathbf{T}_{m 1}(k)+\mathbf{T}_{m m}(k) & \mathbf{T}_{12}(k)-\mathbf{T}_{1 m}(k)-\mathbf{T}_{m 2}(k)+\mathbf{T}_{m m}(k) & \cdots & \mathbf{T}_{1, m-1}(k)-\mathbf{T}_{1 m}(k)-\mathbf{T}_{m, m-1}(k)+\mathbf{T}_{m m}(k) \\
\mathbf{T}_{21}(k)-\mathbf{T}_{2 m}(k)-\mathbf{T}_{m 1}(k)+\mathbf{T}_{m m}(k) & \mathbf{T}_{22}(k)-\mathbf{T}_{2 m}(k)-\mathbf{T}_{m 2}(k)+\mathbf{T}_{m m}(k) & \cdots & \mathbf{T}_{2, m-1}(k)-\mathbf{T}_{2 m}(k)-\mathbf{T}_{m, m-1}(k)+\mathbf{T}_{m m}(k) \\
\vdots & \vdots & \ddots & \vdots \\
\mathbf{T}_{m-1,1}(k)-\mathbf{T}_{m-1, m}(k)-\mathbf{T}_{m 1}(k)+\mathbf{T}_{m m}(k) & \mathbf{T}_{m-1,1}(k)-\mathbf{T}_{m-1, m}(k)-\mathbf{T}_{m 2}(k)+\mathbf{T}_{m m}(k) & \cdots & \mathbf{T}_{m-1, m-1}(k)-\mathbf{T}_{m-1, m}(k)-\mathbf{T}_{m, m-1}(k)+\mathbf{T}_{m m}(k)
\end{array}\right] .
\end{aligned}
$$

Then weighting matrices $\boldsymbol{\Lambda}_{1}(k), \boldsymbol{\Lambda}_{2}(k), \ldots, \boldsymbol{\Lambda}_{m-1}(k) \in R^{n \times n}$ can be determined by solving (20) and $\Lambda_{m}(k)$ can be determined by $\Lambda_{m}(k)=\mathbf{I}-\sum_{i=1}^{m-1} \Lambda_{i}(k)$.

Note. By the processing of the proof, it can be seen that $b_{i j}=$ $c_{j i}, a_{i j} \geq 1+1 /\left(4 b_{i j}\right)+1 /\left(4 c_{i j}\right)$, and the values of parameters $a_{i j}, b_{i j}$, and $c_{i j}$ can be decided from any real numbers $\lambda_{i j}$ and $\mu_{i j}$ as shown in (15). For simplicity, the values can be selected as $\lambda_{i j}=1, \mu_{i j}=1$. Therefore, $a_{i j}=2, b_{i j}=1 / 2, c_{i j}=1 / 2$.

Considering the covariance of fusion result should be less than the covariance of any single measurement, the following is true: $\mathbf{R}(k) \leq \mathbf{R}_{i j}(k)$; that is,

$$
a_{i j} \mathbf{A}(k)+b_{i j} \mathbf{R}_{i i}(k)+c_{i j} \mathbf{R}_{j j}(k) \leq \mathbf{R}_{i j}(k) .
$$

Therefore, the parameters in the theory should satisfy

$$
\begin{gathered}
a_{i j} \geq 1+\frac{1}{4 b_{i j}}+\frac{1}{4 c_{i j}}, \\
\mathbf{A}(k) \leq \frac{\left[\mathbf{R}_{i j}(k)-b_{i j} \mathbf{R}_{i i}(k)-c_{i j} \mathbf{R}_{j j}(k)\right]}{a_{i j}} .
\end{gathered}
$$

\section{Algorithm Validation and Experiment Analysis}

To illustrate the effectiveness of the algorithm, the vehicle system installed two GPS, INS, and other sensors, and the north-east-day geographic coordinates is selected as the navigation coordinate system. The system error equations and measuring equations are

$$
\begin{aligned}
& \dot{\mathbf{x}}(t)=\mathbf{F}(t) \mathbf{x}(t)+\mathbf{G}(t) \mathbf{w}(t), \\
& \mathbf{z}(t)=\mathbf{H}(t) \mathbf{x}(t)+\mathbf{v}(t),
\end{aligned}
$$

where, $\mathbf{x}=\left[\phi_{E}, \phi_{N}, \phi_{U}, \delta V_{E}, \delta V_{N}, \delta V_{U}, \delta L, \delta \lambda, \delta h, \varepsilon_{E}, \varepsilon_{N}\right.$, $\left.\varepsilon_{U}, \nabla_{E}, \nabla_{N}, \nabla_{U}\right]^{T}, \phi_{E}, \phi_{N}$, and $\phi_{U}$ are the misalignment angle for three direction; $\delta V_{N}, \delta V_{E}$, and $\delta V_{U}$ are the east velocity, north velocity, and up velocity error, $\delta L, \delta \lambda$, $\delta h$ are the latitude, longitude, and altitude error, and $\varepsilon_{E}, \varepsilon_{N}$, and $\varepsilon_{U}$ are the drift of the east, north, and up gyroscope. $\nabla_{E}, \nabla_{N}$, and $\nabla_{U}$ are the bias of accelerometer, $\mathbf{F}(t)$ is the system transfer matrix, $\mathbf{z}=\left[V_{E_{-} G P S}-V_{E_{-} I N S}, V_{N_{-} G P S}-V_{N_{-} I N S}, V_{U_{-} G P S}-V_{U_{-} I N S}\right.$, $\left.\lambda_{\mathrm{GPS}}-\lambda_{\mathrm{INS}}, L_{\mathrm{GPS}}-L_{\mathrm{INS}}, h_{\mathrm{GPS}}-h_{\mathrm{INS}}\right]^{T}, \mathbf{G}(t)$ is the system noise matrix, $\mathbf{w}(t)$ is the Gauss noise with zero mean, $\mathbf{H}(t)=\left[\mathbf{0}_{3 \times 3}, \mathbf{I}_{6 \times 6}, \mathbf{0}_{6 \times 6}\right]$ is the measurement matrix, $\mathbf{v}(t)$ is the measurement noise, $\lambda_{\mathrm{GPS}}, L_{\mathrm{GPS}}, h_{\mathrm{GPS}}, \lambda_{\mathrm{INS}}, L_{\mathrm{INS}}$, and $h_{\mathrm{INS}}$ are the position measured by GPS and INS, and $V_{E_{-} G P S}, V_{N_{-} G P S}$, $V_{U_{\text {_GPS }}}, V_{E_{\text {_INS }}}, V_{N_{\text {IINS }}}$, and $V_{U_{\text {_INS }}}$ are the velocity measured by GPS and INS. The state equation is expressed as the following.

(1) Attitude error functions

$$
\begin{gathered}
\dot{\phi}_{E}=\phi_{N}\left[\omega_{i e} \sin L+\frac{V_{E} \tan L}{R_{N}+h}\right] \\
-\phi_{U}\left[\omega_{i e} \cos L+\frac{V_{E}}{R_{M}+h}\right] \\
-\frac{\delta V_{N}}{R_{M}+h}+\frac{V_{N} \delta h}{\left[\left(R_{M}+h\right)^{2}\right]}+\varepsilon_{E}, \\
\dot{\phi}_{N}=-\phi_{E}\left[\omega_{i e} \sin L+\frac{V_{E} \tan L}{R_{N}+h}\right] \\
-\frac{\phi_{U} V_{N}}{R_{M}+h}+\frac{\delta V_{E}}{R_{N}+h} \\
-\omega_{i e} \sin L \delta L-\frac{V_{E} \delta h}{\left[\left(R_{N}+h\right)^{2}\right]}+\varepsilon_{N},
\end{gathered}
$$




$$
\begin{aligned}
\dot{\phi}_{U}= & \phi_{E}\left[\omega_{i e} \cos L+\frac{V_{E}}{R_{N}+h}\right]+\frac{\phi_{N} V_{N}}{R_{M}+h} \\
& +\frac{\tan L \delta V_{E}}{R_{N}+h}-\frac{V_{E} \tan L \delta h}{\left[\left(R_{N}+h\right)^{2}\right]} \\
& +\left[\omega_{i e} \cos L+\frac{V_{E} \sec _{L}^{2}}{R_{N}+h}\right] \delta L+\varepsilon_{U} .
\end{aligned}
$$

(2) Velocity error functions

$$
\begin{aligned}
& \delta \dot{V}_{E}=-f_{U} \phi_{N}+f_{N} \phi_{U}+\left[\frac{V_{N} \tan L-V_{U}}{R_{N}+h}\right] \delta V_{E} \\
& +\left[2 \omega_{i e} \sin L+\frac{V_{E} \tan L}{R_{N}+h}\right] \delta V_{N} \\
& -\left[2 \omega_{i e} \cos L+\frac{V_{E}}{R_{N}+h}\right] \delta V_{U} \\
& +\left[2 \omega_{i e}\left(V_{U} \sin L+V_{N} \cos L\right)+\frac{V_{E} V_{N} \sec ^{2} L}{R_{N}+h}\right] \delta L \\
& +\left[\frac{V_{E} V_{U}-V_{E} V_{N} \tan L}{\left(R_{N}+h\right)^{2}}\right] \delta h+\nabla_{E} \\
& \delta \dot{V}_{N}=-f_{E} \phi_{U}+f_{U} \phi_{E} \\
& -\left[2 \omega_{i e} \sin L+\frac{V_{E} \tan L}{R_{N}+h}\right] \delta V_{E} \\
& -\left[\frac{V_{U}}{R_{M}+h}\right] \delta V_{N}-\left[\frac{V_{N}}{R_{M}+h}\right] \delta V_{U} \\
& -\left[2 \omega_{i e} V_{E} \cos L+\frac{V_{E}^{2} \sec ^{2} L}{R_{N}+h}\right] \delta L \\
& +\left[\frac{V_{U} V_{N}}{\left(R_{M}+h\right)^{2}}+\frac{V_{E} \tan L}{\left(R_{N}+h\right)^{2}}\right] \delta h+\nabla_{N}, \\
& \delta \dot{V}_{U}=f_{E} \phi_{N}-f_{N} \phi_{E} \\
& +\left[2 \omega_{i e} \cos L+\frac{V_{E}}{R_{N}+h}\right] \delta V_{E} \\
& +\left[\frac{2 V_{N}}{R_{M}+h}\right] \delta V_{N}-2 \omega_{i e} V_{E} \sin L \delta L \\
& -\left[\frac{V_{N}}{\left(R_{M}+h\right)^{2}}+\frac{V_{E}}{\left(R_{N}+h\right)^{2}}\right] \delta h+\nabla_{U} .
\end{aligned}
$$

(3) Position error functions

$$
\begin{aligned}
\delta \dot{L}= & {\left[\frac{1}{R_{M}+h}\right] \delta V_{N}-\left[\frac{V_{N}}{\left(R_{M}+h\right)^{2}}\right] \delta h } \\
\delta \dot{\lambda}= & {\left[\frac{\sec L}{R_{N}+h}\right] \delta V_{E} } \\
& +\left[\frac{V_{E} \sec L \tan L}{R_{N}+h}\right] \delta L \\
& -\left[\frac{V_{E} \sec L}{\left(R_{N}+h\right)^{2}}\right] \delta h \\
\delta \dot{h}= & \delta V_{U} .
\end{aligned}
$$

(4) Error functions of inertial device

$$
\begin{array}{ccc}
\dot{\varepsilon}_{E}=0, & \dot{\varepsilon}_{N}=0, & \dot{\varepsilon}_{U}=0, \\
\dot{\nabla}_{E}=0, & \dot{\nabla}_{N}=0, & \dot{\nabla}_{U}=0,
\end{array}
$$

where $R_{M}=R\left(1-2 e+3 e \sin ^{2} L\right), R_{N}=R\left(1+e \sin ^{2} L\right)$ are the radius of curvature for the meridian plane and the prime vertical plane. $R$ is the length of major semiaxis, $e$ is the eccentricity ratio of the earth, the subscripts $E, N, U$ denote the directions of earth, north, and up, $L, \lambda, h$ denote longitude, latitude, and altitude, $\omega_{i e}=15.04107^{\circ} / \mathrm{h}$ is the rotational velocity in the earth inertial space, and $g=9.8 \mathrm{~m} / \mathrm{s}^{2}$ is the gravitational acceleration.

Shown in Figure 1 is the measurement information of attitude and acceleration of the vehicle during the experiment.

Without loss of generality, the measurement noise of different sensors from the vehicle system is affected by uncertainty term.

According to formula (24), the parameters in the theory are selected as $a_{i j}=2, b_{i j}=1 / 2, c_{i j}=1 / 2$, and $\mathbf{A}(k)=$ $0.5\left(\mathbf{R}_{i j}(k)-0.5 \mathbf{R}_{i i}(k)-0.5 \mathbf{R}_{j j}(k)\right)$, where $\mathbf{R}_{i j}(k)$ are determined by the accuracy of the sensors. The equivalent measurement value, obtained by formula (6), is substituted into the system equations (24) and then the longitude, latitude, and altitude of the vehicle will be obtained after the filtering. The results are shown in Figures 2, 3, and 4.

The above figures show that the estimated value of longitude and latitude is matched with the measured value very well, while the difference of the altitude is relatively large. This is due to the distance from north-south direction on the sphere changing 111 kilometers when the latitude changes one degree. That is to say, the distance will change 30.9 meters when latitude changes one second. The proportion of transfer relationship for two units for longitude is similar with latitude, while the proportion for longitudinal is not the constant; it is also related with latitude of the position, but the basic proportions will not vary too much. Therefore, when the unit of longitude and latitude is degrees, the difference between estimated and measured value is very small.

In order to illustrate uncertainty effects on the fusion error of longitude, latitude, and altitude, Figures 5, 6, and 7 

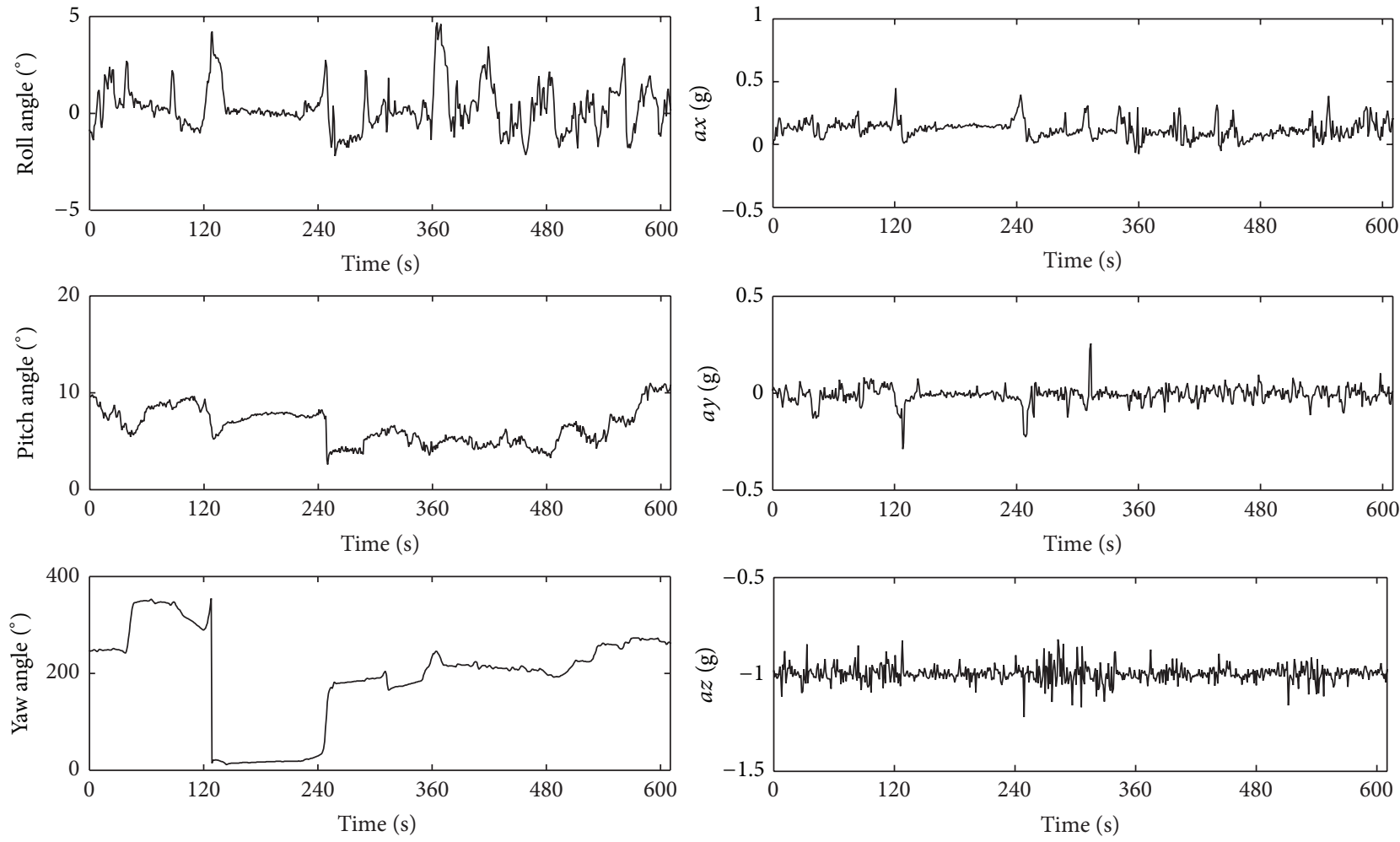

FIGURE 1: Response of attitude and acceleration information of the vehicle.

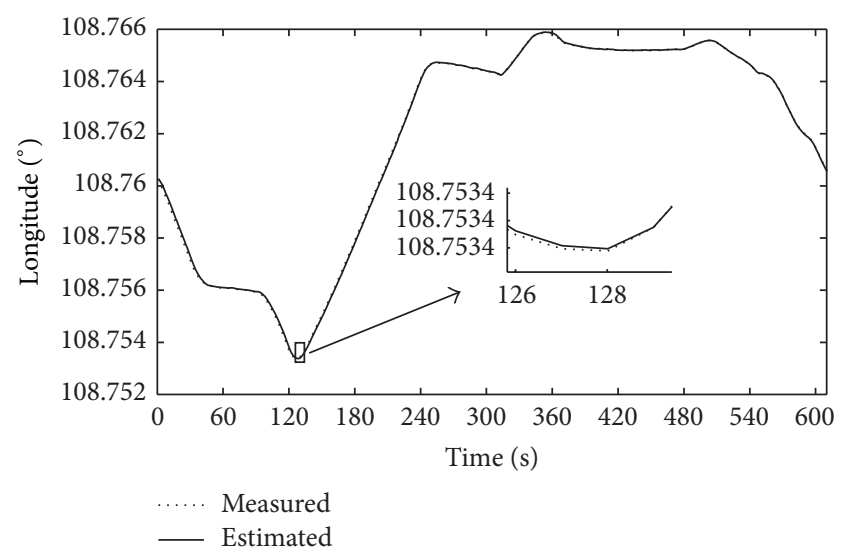

FIGURE 2: Measured and estimated value of the longitude.

show the fusion error curves of vehicle position, whose unit is meters.

From the introduction part, it can be seen that many fusion algorithms are studied with the assumption that the measurement noises are independent with Gaussian noise distribution. In order to compare the results of this paper with the independent noise assumption, the fusion error curves with independent noise assumption are also shown in Figures 5 to 7.

From Figures 5 to 7 , it can be seen that for the same measurement information, the fusion error of longitude, altitude, and altitude with uncertainty noise assumption is

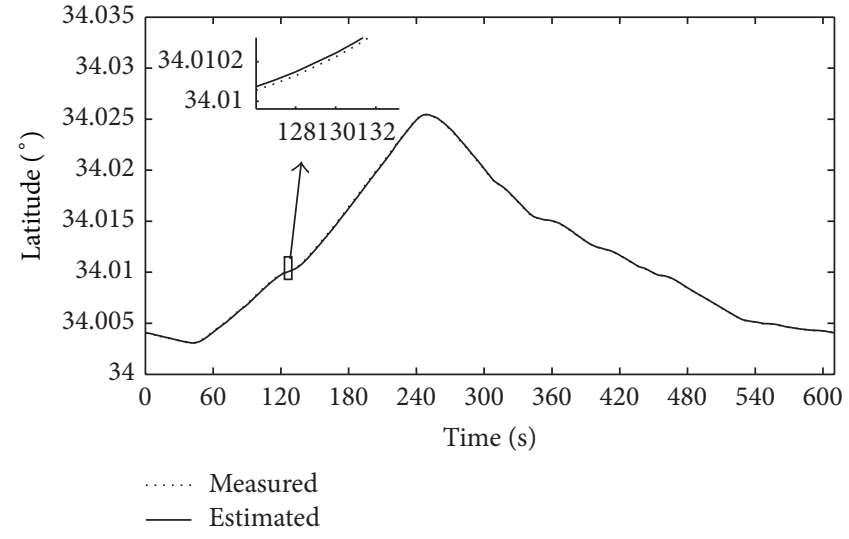

FIgURE 3: Measured and estimated value of the latitude.

better than the independent noise assumption. It can be also observed that the difference of altitude error curves is much more obvious than the longitude and latitude errors with two assumptions. This is because that the changes in longitude and latitude are mainly affected by the longitudinal and lateral movement of the vehicle, and change of altitude is mainly affected by the vertical movement of the vehicle. Because the uncertainty factors in the experiment are mainly from the vehicle vibration during the driving, the effects of vibration to longitudinal and lateral motion is less than the vertical motion. This conclusion illustrates that the uncertainty factors do really exist for the actual system especially 


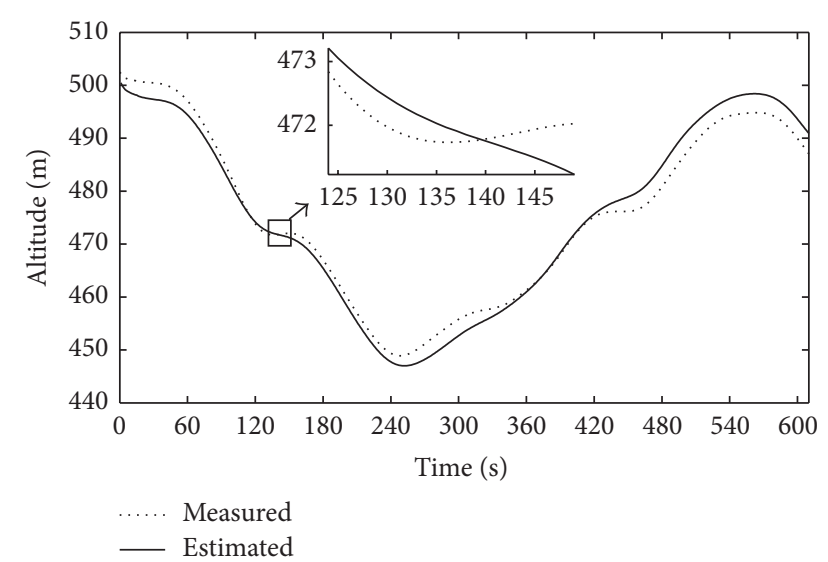

FIGURE 4: Measured and estimated value of the latitude.

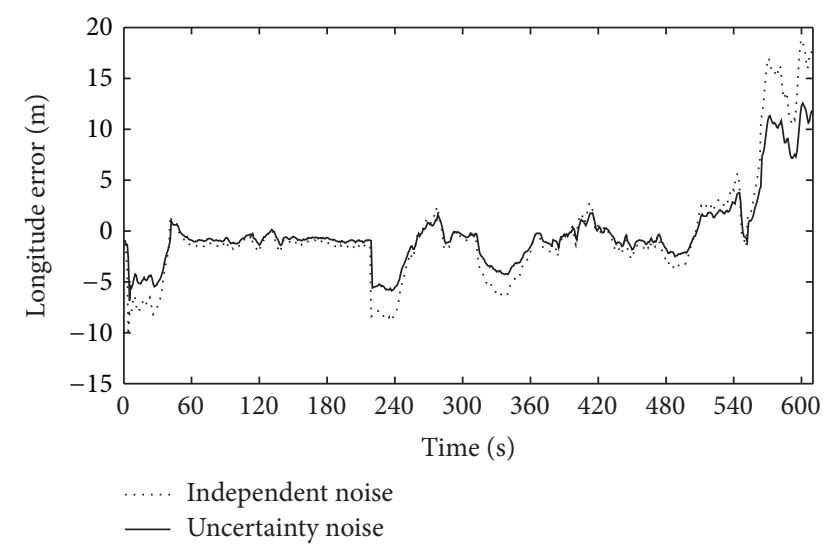

FIGURE 5: Longitude error with independent and uncertainty noise.

when multiple sensors are installed on the same platform. The vibration of platform makes the measurement noises of different sensors are intercorrelated and the statistical characteristic of the noise is unknown. Taking into account the uncertainty factors of measurement noise in the actual system can help to improve the positioning accuracy of the vehicle.

\section{Conclusions}

For the actual system with multiple sensors to detect the same state vector, this paper proposed an extended Kalman filter method based on multisensor fusion algorithm with uncertainty disturbance. This method can deal with the problem of uncertainty effects of measurement noise by fusing the measurement information and filtering the system equation.

What is more, the accurate fusion results can be obtained just by one time filtering which reduces the calculation. The fusion result is verified by the GPS/INS navigation system, which shows that the algorithm proposed here considering the uncertainty influences for the system enhances the positioning accuracy for actual system.

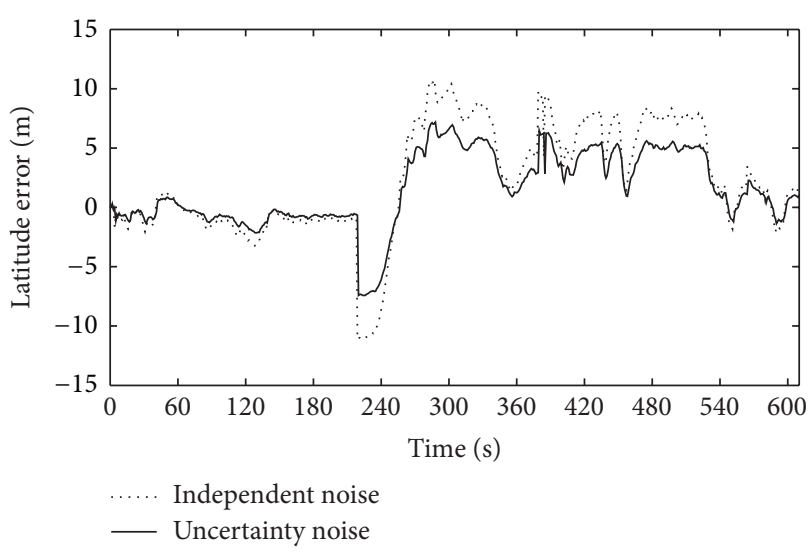

FIGURE 6: Latitude error with independent and uncertainty noise.

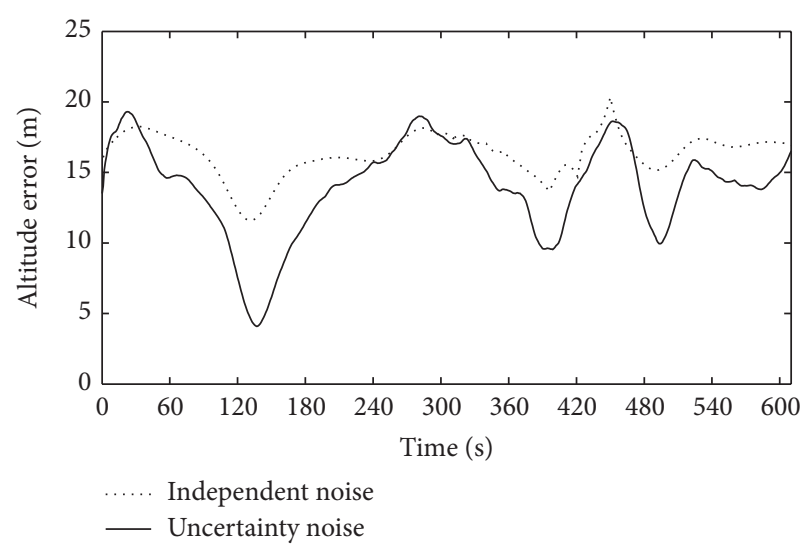

FIGURE 7: Altitude error with independent and uncertainty noise.

\section{Conflict of Interests}

The authors declare that there is no conflict of interests regarding the publication of this paper.

\section{Acknowledgments}

This research is supported by the National Natural Science Foundation of China (no. 50974103) and Educational Commission of Shaanxi Province of China (2013JK0858).

\section{References}

[1] D.-J. Jwo and S.-H. Wang, "Adaptive fuzzy strong tracking extended Kalman filtering for GPS navigation," IEEE Sensors Journal, vol. 7, no. 5, pp. 778-789, 2007.

[2] A. Noureldin, A. El-Shafie, and M. Bayoumi, "GPS/INS integration utilizing dynamic neural networks for vehicular navigation," Information Fusion, vol. 12, no. 1, pp. 48-57, 2011.

[3] G. A. Einicke, G. Falco, and J. T. Malos, "Bounded constrained filtering for GPS/INS integration," IEEE Transactions on Automatic Control, vol. 58, no. 1, pp. 125-133, 2013.

[4] J. Rezaie, B. Moshiri, B. N. Araabi, and A. Asadian, "GPS/INS integration using nonlinear blending filters," in Proceedings of 
the SICE Annual Conference, pp. 1674-1680, IEEE, Takamatsu, Japan, September 2007.

[5] F. Caron, E. Duflos, D. Pomorski, and P. Vanheeghe, "GPS/IMU data fusion using multisensor Kalman filtering: introduction of contextual aspects," Information Fusion, vol. 7, no. 2, pp. 221230, 2006.

[6] I. Arasaratnam, S. Haykin, and T. R. Hurd, "Cubature Kalman filtering for continuous-discrete systems: theory and simulations," IEEE Transactions on Signal Processing, vol. 58, no. 10, pp. 4977-4993, 2010.

[7] M. Šimandl and J. Duník, "Derivative-free estimation methods: new results and performance analysis," Automatica, vol. 45, no. 7, pp. 1749-1757, 2009.

[8] Q. Gan and J. H. Chris, "Comparison of two measurement fusion methods for kalman-filter-based multisensor data fusion," IEEE Transactions on Aerospace and Electronic Systems, vol. 37, no. 1, pp. 273-280, 2001.

[9] J. Liu and B. Cai, "Adaptive algorithm of information fusion for integrated navigation," Journal of Beijing Jiaotong University, vol. 32, no. 2, pp. 88-93, 2008.

[10] Z.-L. Deng and Y. Gao, "Fast information fusion Kalman filter," Control and Decision, vol. 20, no. 1, pp. 27-31, 2005.

[11] C. Cappelle, D. Pomorski, and Y. Yang, "GPS/INS data fusion for land vehicle localization," in Proceedings of the IMACS Multiconference on Computational Engineering in Systems Applications, vol. 1, pp. 21-27, Beijing, China, 2006.

[12] N. A. Carlson, "Federated square root filter for decentralized parallel processes," IEEE Transactions on Aerospace and Electronic Systems, vol. 26, no. 3, pp. 517-525, 1990.

[13] Z. Deng and R. Qi, "Multi-sensor information fusion suboptimal steady-state Kalman filter," Chinese Science Abstracts, vol. 6, no. 2, pp. 183-184, 2000.

[14] A. Giremus, E. Grivel, and F. Castanie, "Is $H_{\infty}$ filtering relevant for correlated noises in GPS navigation?" in Proceedings of the 16th International Conference on Digital Signal Processing (DSP '09), pp. 1-6, IEEE, July 2009.

[15] X. Wang, Y. Liang, Q. Pan, and F. Yang, "A Gaussian approximation recursive filter for nonlinear systems with correlated noises," Automatica, vol. 48, no. 9, pp. 2290-2297, 2012.

[16] S.-L. Sun, "Multi-sensor optimal information fusion Kalman filters with applications," Aerospace Science and Technology, vol. 8, no. 1, pp. 57-62, 2004.

[17] S. Sun and Z. Deng, "Multi-sensor optimal information fusion Kalman filter," Automatica, vol. 40, no. 6, pp. 1017-1023, 2004.

[18] J. Hong, Z. Hong, and Y. Hong, "Fusion algorithm of correlated local estimates for federated filter," in Proceedings of the $3 \mathrm{rd}$ Asian Control Conference, pp. 1428-1433, Shanghai, China, 2000.

[19] H. Z. Qiu, H. Y. Zhang, and H. Jin, "Fusion algorithm of correlated local estimates," Aerospace Science and Technology, vol. 8, no. 7, pp. 619-626, 2004. 


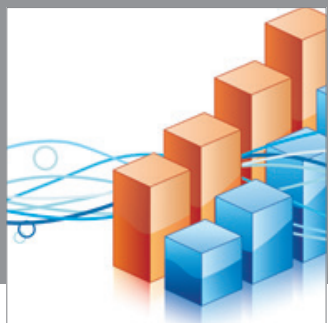

Advances in

Operations Research

mansans

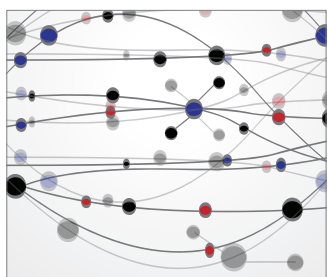

The Scientific World Journal
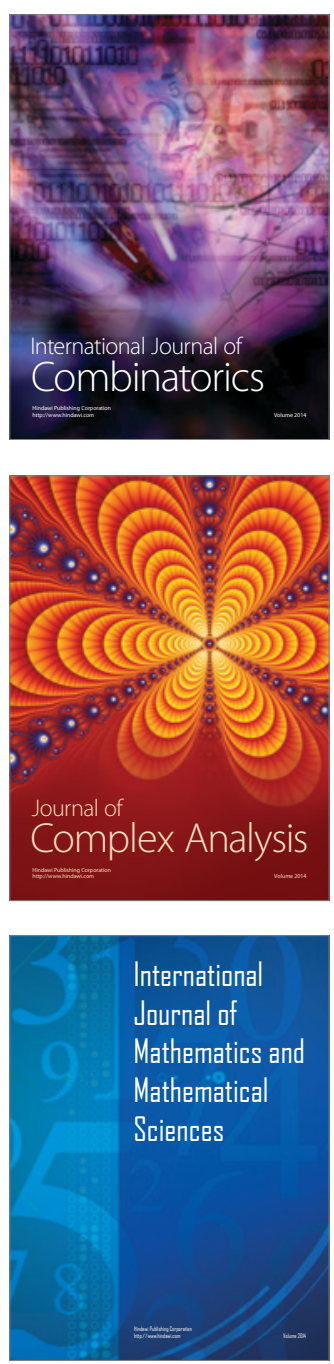
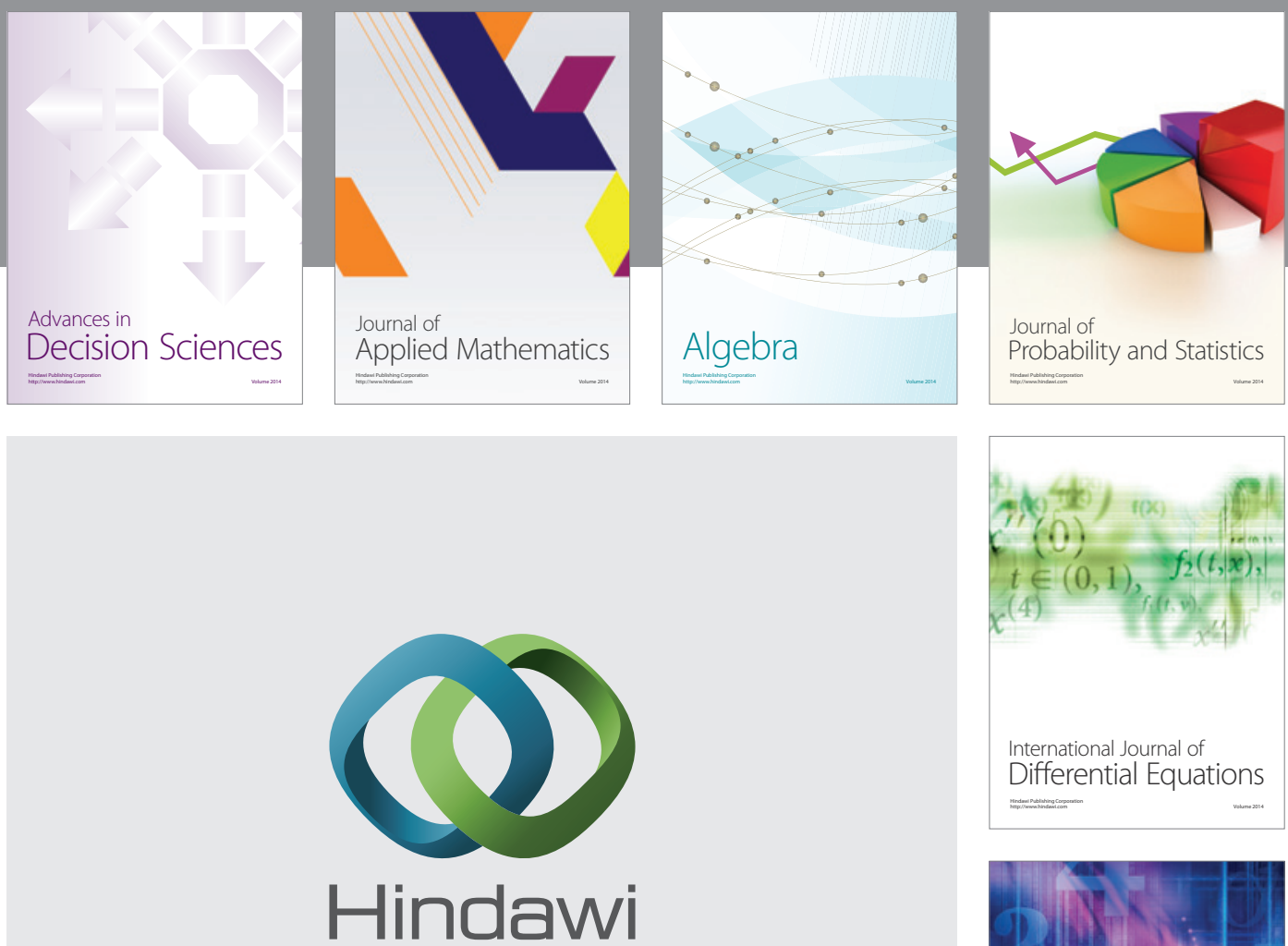

Submit your manuscripts at http://www.hindawi.com
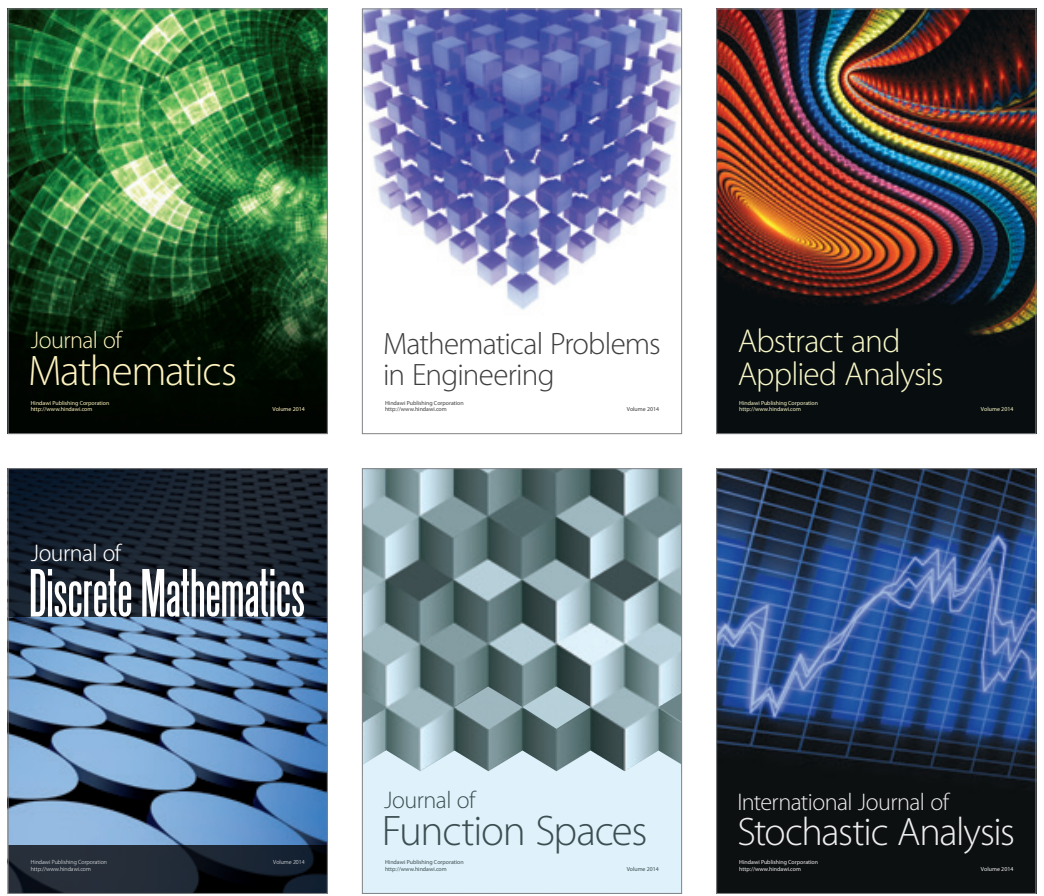

Journal of

Function Spaces

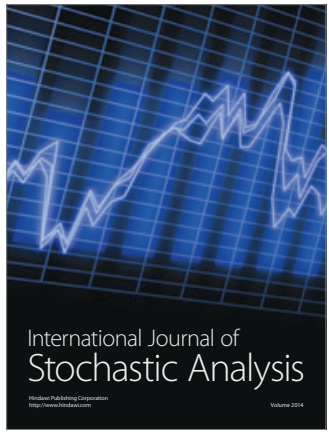

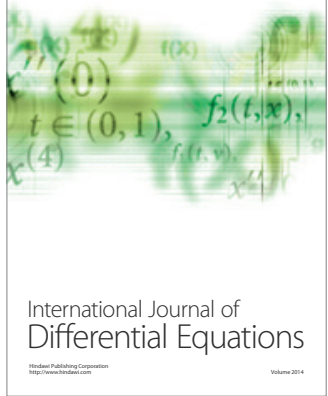
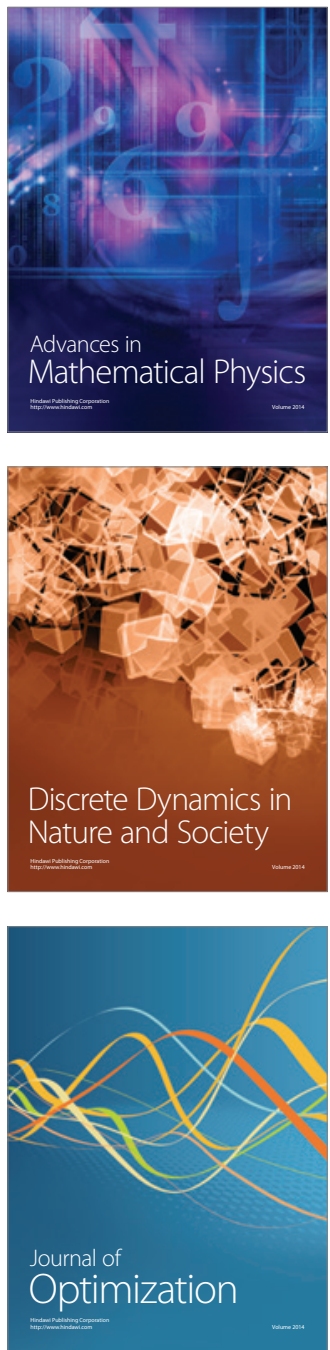